\title{
Association Between Lipid Accumulation Product and Target Organ Damage in Elderly Population: The Northern Shanghai Study
}

\author{
Song Zhao \\ Zhongyuan Ren \\ Shikai Yu \\ Chen Chi $\mathbb{D}$ \\ Jiamin Tang \\ Rusitanmujiang Maimaitiaili \\ Jiadela Teliewubai \\ Jiaxin $\mathrm{Li}$ \\ Yawei Xu \\ Yi Zhang \\ Department of Cardiology, Shanghai \\ Tenth People's Hospital, School of \\ Medicine, Tongji University, Shanghai, \\ 200072, People's Republic of China
}

Correspondence: Yi Zhang; Yawei Xu Department of Cardiology, Shanghai Tenth People's Hospital, School of Medicine, Tongji University, 30I Yanchang Road, Shanghai, 200072, People's Republic of China

Tel/Fax $+86 \quad 189 \mid 7686332$

Email yizshcn@gmail.com;

xuyawei@tongji.edu.cn
Introduction: Lipid accumulation product (LAP), calculated from waist circumference (WC) and triglycerides (TG), is a novel index that correlates cardiovascular disease. We aimed to investigate the relationship between LAP and target organ damage (TOD) in elderly Chinese community-dwelling individuals.

Materials and Methods: We enrolled 3363 participants whose age was $\geq 65$ years old. TOD, including left ventricular hypertrophy (LVH), arterial stiffness (AS), lower extremity atherosclerotic (LEA), micro-albuminuria (MAU) and chronic kidney disease (CKD), was measured using standard methods. LAP was calculated as $(\mathrm{WC}-65) \times \mathrm{TG}$ in men and $(\mathrm{WC}-$ $58) \times$ TG in women. Both quartiles and continuation of LAP were analyzed.

Results: Age-sex adjusted partial correlation analysis showed that LAP was significantly associated with CVD risk factors. With the first quartile (Q1) as a reference, in univariate logistic regression, the fourth quartile (Q4) of LAP was associated with all TOD. In multivariate model, Q4 of LAP was only associated with an increased risk of AS (odds ratio (OR) $=1.88,95 \%$ confidence interval $\left.(\mathrm{CI}): 1.37-2.58, \mathrm{P}_{\text {for trend }}<0.001\right)$, MAU $(\mathrm{OR}=1.33,95 \%$ CI: $\left.1.01-1.75, \mathrm{P}_{\text {for trend }}=0.02\right)$ and $\mathrm{CKD}\left(\mathrm{OR}=2.39,95 \% \mathrm{CI}: 1.39-4.12, \mathrm{P}_{\text {for trend }}<0.001\right)$. But, Q4 of LAP was not associated with an increased risk of LVH (OR $=1.19,95 \% \mathrm{CI}$ : $\left.0.85-1.65, \mathrm{P}_{\text {for trend }}=0.25\right)$ or LEA (OR $=0.87,95 \%$ CI: $\left.0.58-1.29, \mathrm{P}_{\text {for trend }}=0.96\right)$. Similar associations were found when analyzed continuously.

Conclusion: The novel metabolic parameter LAP is significantly and independently associated with an increased risk of arterial stiffness, chronic kidney disease and microalbuminuria in Chinese community-dwelling elderly individuals.

Keywords: lipid accumulation product, target organ damage, elderly

\section{Introduction}

Obesity indicates the accumulation of body fat, which is closely related to health and strongly associated with cardiovascular disease (CVD). Meanwhile, body fat can accumulate either as subcutaneous adipose tissue (SAT) or visceral adipose tissue (VAT). Studies have shown that VAT rather than SAT was more closely correlated with CVD. ${ }^{1,2}$ Therefore, measurement of obesity plays an important role in management of CVD. Hitherto, several indexes have been proposed and routinely used, including body mass index (BMI, $\mathrm{kg} / \mathrm{m}^{2}$ ) and waist circumference (WC). But neither could distinguish accumulation of VAT from SAT. ${ }^{3}$ Computed tomography $(\mathrm{CT})^{4}$ and magnetic resonance imaging (MRI) ${ }^{5}$ are the gold standard for measuring visceral fat, but they are inconvenient and expensive. Thus, lipid 
accumulation product (LAP)-a metabolic index combining WC and triacylglycerol (TG)-has been suggested as a marker of central lipid accumulation. ${ }^{6-8}$

Kahn first described LAP as better than BMI for recognizing cardiovascular risk. ${ }^{6,9}$ LAP has been proven to be a powerful marker of metabolic syndrome, ${ }^{10-12}$ as well as a predictor of diabetes. ${ }^{5,13}$ Studies showed that LAP was higher in patients with diabetes ${ }^{9}$ and closely related to insulin resistance and glucose metabolic disorder. ${ }^{8,14}$ Following studies revealed the association of LAP with all-cause mortality in patients with high cardiovascular risk. ${ }^{15}$ Based on current evidence, LAP may play a role in evaluating body fat and is a powerful predictor of CVD.

Among various risk factors, several studies have proved that obesity predisposes TOD. ${ }^{16-18}$ As a novel and effective measure of obesity, LAP has been proven to be associated with TOD-like renal impairment. ${ }^{19}$ Nevertheless, studies concerning the application of the LAP to target organ damage (TOD) are very limited. Therefore, we aim to investigate the association between LAP and TOD in our large Chinese community-dwelling elderly individuals, in order to regard the LAP application in elderly population.

\section{Materials and Methods}

\section{Participants}

Northern Shanghai Study (NSS) was signed at ClinicalTrials.gov (Identifier: NCT02368938). The protocol of NSS had been published previously. ${ }^{20}$ We randomly selected 10 neighborhoods in the northern region of Shanghai (Zhabei district and Putuo district) by computer-generated list of communities. The recruitment strategies include: (1) posting study recruitment files in the neighborhood committees and community hospitals; (2) according to the health file, community hospitals recruit the potential participants by telephone; (3) hand out recruitment flyers directly to the potential participants. When individuals express their intentions in participating in the study, the individual will be adequately informed, and they will sign the consent form. We included participants who met the following criteria. (1) Age more than 65 years old; (2) Residents of Shanghai in northern urban communities and (3) Informed consent was signed and long-term follow-up can be performed. The exclusion criteria were follows: (1) Class IV of New York Heart Association (NYHA) functional or class 5 of CKD; (2) A malignant tumor; (3) Stroke history in the previous 3 months. 3590 residents were invited to participate, and 3363 (93.7\%) participants who agreed to participate in the study enrolled from June 2014 to August 2019.

\section{Social, Clinical, and Biological Parameters}

All participants received standard questionnaires to collect relevant information, such as gender, age, education level, smoking habits, drinking habits, history of hypertension, history of diabetes, history of CVD and so on. Physical examination was completed by professionals. Body height and body weight are measured by disrobe, removing shoes and standing straight. A flexible rule was used to measure WC and hipline. BMI was computed by dividing body weight $(\mathrm{kg})$ by the squared body height $\left(\mathrm{m}^{2}\right)$. After 5 minutes rests, blood pressure was measured three times by a semiautomatic oscillometric device (Omron, Japan) at 2-minute intervals, and the average of the three measurements was calculated. Hypertension is defined by the average blood pressure at which systolic and/or diastolic blood pressure (SBP/DBP) is greater than 140/90 $\mathrm{mmHg}$. The subjects with the current use of antihypertensive medication are also defined as Hypertension. Venous blood samples were obtained after an overnight fast. Morning spot urine sample was obtained from morning urine. All samples were analyzed in the Department of Laboratory Medicine of Shanghai Tenth People's Hospital by professional technicians. LAP was computed as ((WC [cm]-65) $\times(\mathrm{TG}[\mathrm{mmol} / \mathrm{L}])$ in men, or $((\mathrm{WC}[\mathrm{cm}]-58) \times \mathrm{TG}[\mathrm{mmol} /$ L]) in women.

\section{Measurement and Definition of TOD}

We used MyLab $30 \mathrm{CV}$ machine (ESAOTE SPA) to perform cardiac ultrasonography. Both left ventricular internal diameter (LVIDd), septal (SWTd) and posterior wall thickness at the end diastole (PWTd) were measured, respectively. The formula, $0.8 \times\{1.04 \times[$ (LVIDd + PWTd + SWTd $\left.)^{3}-(\text { LVIDd })^{3}\right]+0.6$, was used to calculated left ventricular mass (LVM). Left ventricular hypertrophy (LVH) was defined as $L V M I \geq 115 \mathrm{~g} / \mathrm{m}^{2}$ for men or $\mathrm{LVMI} \geq 95 \mathrm{~g} / \mathrm{m}^{2}$ for women.

Applanation tonometry (SphygmoCor, AtCor Medical, Sydney, Australia) was used to measure carotid-femoral pulse wave velocity (cf-PWV) after rest at least 10 minutes. Arterial stiffness (AS) was defined as cf$\mathrm{PWV}>10 \mathrm{~m} / \mathrm{s}$.

Ankle-brachial index (ABI) was automatically calculated by a VP1000 system (Omron, Kyoto, Japan) after 
rest for at least 10 minutes. Lower extremity atherosclerotic (LEA) was defined as $\mathrm{ABI}<0.9$.

Blood and urine samples were examined in the center of Shanghai Tenth People's Hospital at morning. Microalbuminuria (MAU) was defined as urinary albuminto-creatinine ratio (UACR) $>30 \mathrm{mg} / \mathrm{g}$. Modification of Diet in Renal Disease formula was used to compute estimated glomerular filtration rate (eGFR). ${ }^{21} \mathrm{CKD}$ was defined as $\mathrm{eGFR} \leq 60 \mathrm{~mL} / \mathrm{min}$ per $1.73 \mathrm{~m}^{2}$.

\section{Statistical}

Statistical analysis was conducted using SAS software version 9.4 (SAS Institute, Inc., Cary, North Carolina, USA). All subjects were categorized into four groups according to the quartiles of LAP. Mean \pm standard deviation (SD) was used to present continuous variables. Categorical variables were presented as numbers with proportions. Differences of parameters between the four groups were compared using the ANOVA test for continuous variables and $\mathrm{CMH}$ test for categorical variables. We used age-sex adjusted partial-correlation to analyse the associations of LAP with conventional metabolic parameters. Logistic regression model was used to explore the relationship between the different quartiles of LAP and TOD risk, with the lowest LAP quartile as reference. Both crude model and adjusted model (adjusted for age (years), sex $(1=$ male, $0=$ female $)$, BMI $\left(\mathrm{kg} / \mathrm{m}^{2}\right)$, smoking habit $(0=$ no, $1=$ yes $)$, hypertension $(0=$ no, $1=$ yes $)$, use of antihypertensive $(0=$ no, $1=$ yes $)$, family history of premature CVD $(0=$ no, $1=$ yes $)$, diabetes $(0=$ no, $1=$ yes $)$, Highdensity lipoprotein cholesterol (HDL-C, $\mathrm{mmol} / \mathrm{L}$ ) and Low-density lipoprotein cholesterol (LDL-C, mmol/L)) were applied. We also showed the effect estimate of 1-SD increases in LAP. C-statistic for LAP, WC and TG in univariate logistic regression were performed. Setting $\mathrm{P}$ value $<0.05$ is considered significant for all the analysis.

\section{Results}

As shown in Table 1, We found that mean ABI (Q1=1.04, $\mathrm{Q} 2=1.04, \mathrm{Q} 3=1.02, \mathrm{Q} 4=1.00)$, mean LVMI (Q1=82.7, $\left.\mathrm{Q} 2=86.7, \quad \mathrm{Q} 3=89.5, \quad \mathrm{Q} 4=92.3 \mathrm{~g} / \mathrm{m}^{2}\right)$, mean $\mathrm{cf}-\mathrm{PWV}$ $(\mathrm{Q} 1=8.81, \quad \mathrm{Q} 2=9.18, \quad \mathrm{Q} 3=9.42, \quad \mathrm{Q} 4=9.91 \mathrm{~m} / \mathrm{s})$, mean eGFR (Q1=87.3, Q2=86.5, Q3=84.5, Q4=82.1, $\mathrm{mL} / \mathrm{min} /$ $\left.1.73 / \mathrm{m}^{2}\right)$, mean Log (UACR) (Q1=3.34, Q2=3.33, $\mathrm{Q} 3=3.45, \mathrm{Q} 4=3.70 \mathrm{mg} / \mathrm{g}$ ) were different among the four groups. LAP was significantly correlated with BMI, SBP, DBP, FPG, TC and LDL-C (all $\mathrm{P}<0.05)$ after adjustment for age and sex. However, there was no correlation between HDL-C and LAP $(\mathrm{P}=0.09)$ after adjustment (Table 2).

As shown in Table 3, in univariate logistic regression, comparing Q2, Q3, Q4 level with Q1 level of LAP, odds ratio $(\mathrm{OR})$ of $\mathrm{LVH}$ was 1.31 (95\% confidence interval [95\% CI]: 1.03-1.67), 1.70 (95CI\%: 1.34-2.15) and 2.22 (95\% CI: 1.76-2.79) $\left(\mathrm{P}_{\text {for trend }}<0.001\right)$ respectively; OR of AS was 1.47 (95CI\%: 1.18-1.83), 1.59 (95CI\%: 1.28$1.98)$ and 2.37 (95CI\%: 1.91-2.93) $\left(\mathrm{P}_{\text {for trend }}<0.001\right)$ respectively; OR of LEA was 0.84 (95CI\%: 0.61-1.15), 1.19 (95CI\%:0.89-1.59) and 1.69 (95CI\%: 1.28-2.22) $\left(\mathrm{P}_{\text {for trend }}<0.001\right)$ respectively; OR of MAU was 0.95 (95CI\%: 0.78-1.15), 1.13 (95CI\%: 0.93-1.36) and 1.49 (95CI\%: 1.22-1.80) ( $\left.\mathrm{P}_{\text {for trend }}<0.001\right)$ respectively; OR of CKD was 0.83 (95CI\%: 0.58-1.20), 1.11 (95CI\%:

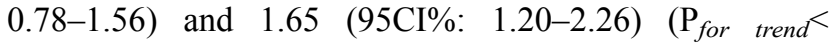
$0.001)$ respectively.

As shown in Table 4, after adjustment for multiple variables (age (years), sex $(1=$ male, $0=$ female $)$, BMI $(\mathrm{kg} /$ $\left.\mathrm{m}^{2}\right)$, smoking habit $(0=$ no, $1=$ yes $)$ hypertension $(0=$ no, $1=$ yes $)$, use of antihypertensive $(0=$ no, $1=$ yes $)$, family history of premature $\operatorname{CVD}(0=$ no, $1=$ yes $)$, diabetes $(0=$ no, $1=$ yes $)$, HDL-C $(\mathrm{mmol} / \mathrm{L})$ and LDL-C (mmol/L)), comparing Q2, Q3, Q4 level with Q1 level of LAP, OR of LVH was 1.02 (95CI\%: 0.78-1.34), 1.10 (95CI\%: 0.82$1.47)$ and 1.19 (95CI\%: 0.85-1.65) $\left(\mathrm{P}_{\text {for trend }}=0.25\right)$ respectively; OR of AS was 1.31 (95CI\%: 1.02-1.70), 1.39 (95CI\%: 1.05-1.85) and 1.88 (95CI\%: 1.37-2.58) $\left(\mathrm{P}_{\text {for trend }}<0.001\right)$ respectively; OR of LEA was 0.64 (95CI\%: 0.45-0.90), 0.79 (95CI\%: 0.55-1.12) and 0.87 (95CI\%: 0.58-1.29) $\left(\mathrm{P}_{\text {for trend }}=0.96\right)$ respectively; OR of MAU was 0.91 (95CI\%: 0.73-1.13), 1.06 (95CI\%: 0.84$1.36)$ and 1.33 (95CI\%: 1.01-1.75) $\left(\mathrm{P}_{\text {for trend }}=0.02\right)$ respectively; OR of CKD was 1.35 (95CI\%: 0.83-2.19), 1.78 (95CI\%: 1.08-2.92) and 2.39 (95CI\%: 1.39-4.12) $\left(\mathrm{P}_{\text {for trend }}<0.001\right)$ respectively.

As shown in Figure 1, when LAP was analyzed continuously, 1-SD increment of LAP and associated with LVH $(\mathrm{OR}=1.29$, 95\% CI: 1.19-1.39, $\mathrm{P}<0.001)$, AS $(\mathrm{OR}=1.35$, 95\% CI: $1.25-1.46, \mathrm{P}<0.001)$, LEA $(\mathrm{OR}=1.21$, 95\% CI: 1.11-1.32, $\mathrm{P}<0.001)$, MAU (OR=1.25, 95\% CI: 1.16-1.35, $\mathrm{P}<0.001)$ and $\mathrm{CKD} \quad(\mathrm{OR}=1.31,95 \% \quad \mathrm{CI}:$ 1.20-1.44, $\mathrm{P}<0.001$ ). As shown in Figures 2, 1-SD increment of LAP was associated with increased risk of $\mathrm{AS}(\mathrm{OR}=1.23,95 \%$ CI: $1.10-1.36, \mathrm{P}<0.001)$, MAU (OR=1.21, 95\% CI: 1.10 1.35, $\mathrm{P}<0.001)$ and $\mathrm{CKD}(\mathrm{OR}=1.20,95 \% \mathrm{CI}: 1.06-1.36$, $\mathrm{P}=0.005$ ) after adjustment for covariates, but not LVH 
Table I. Characteristics of Participants

\begin{tabular}{|c|c|c|c|c|c|c|}
\hline & $\begin{array}{l}\text { Overall } \\
(n=3363)\end{array}$ & $\begin{array}{c}\text { QI }(\text { LAP }<21.45, \\
n=874)\end{array}$ & $\begin{array}{c}\text { Q2 } \\
(21.45 \leq \text { LAP<35.48, } \\
n=827)\end{array}$ & $\begin{array}{c}\text { Q3 } \\
(35.28 \leq \mathrm{LAP}<55.48, \\
n=832)\end{array}$ & $\begin{array}{c}\text { Q4(LAP } \geq 55.48, \\
n=830)\end{array}$ & $\mathbf{P}$ \\
\hline Age (years) & $71.1 \pm 6.0$ & $71.1 \pm 6.1$ & $71.0 \pm 5.8$ & $71.0 \pm 6.1$ & $71.3 \pm 6.0$ & 0.79 \\
\hline Male, n (\%) & I467(43.63) & $472(54.00)$ & $389(47.04)$ & $319(38.34)$ & $287(34.58)$ & $<0.001$ \\
\hline Hypertension, n (\%) & $2220(66.01)$ & $459(52.52)$ & $538(65.05)$ & $596(71.63)$ & $627(75.54)$ & $<0.001$ \\
\hline Diabetes mellitus, n (\%) & $756(22.48)$ & $148(16.93)$ & $169(20.44)$ & $175(21.03)$ & $264(31.8 I)$ & $<0.001$ \\
\hline Smoking habit, n (\%) & $833(24.77)$ & $249(28.49)$ & $215(26.00)$ & $\mid 79(2|.5|)$ & $190(22.89)$ & 0.004 \\
\hline Family history of premature CVD, $\mathrm{n}(\%)$ & $722(21.47)$ & $176(20.14)$ & $183(22.13)$ & $170(20.43)$ & $193(23.25)$ & 0.36 \\
\hline $\mathrm{SBP}(\mathrm{mmHg})$ & $135.5 \pm 17.2$ & $130.5 \pm 17.1$ & $135.4 \pm 16.7$ & $136.8 \pm|6|$. & $139.5 \pm 17.5$ & $<0.001$ \\
\hline $\mathrm{DBP}(\mathrm{mmHg})$ & $79.2 \pm 9.6$ & $77.0 \pm 9.0$ & $78.8 \pm 9.2$ & $80.7 \pm 9.9$ & $80.6 \pm 10.1$ & $<0.001$ \\
\hline$W C(\mathrm{~cm})$ & $86.6 \pm 9.9$ & $77.5 \pm 7.9$ & $85.0 \pm 6.6$ & $89.6 \pm 7.1$ & $94.5 \pm 8.6$ & $<0.001$ \\
\hline BMI $(\mathrm{kg} / \mathrm{m} 2)$ & $24.6 \pm 3.6$ & $21.2 \pm 2.8$ & $23.5 \pm 2.5$ & $25.0 \pm 2.8$ & $26.7 \pm 3.7$ & $<0.001$ \\
\hline FPG (mmol/L) & $5.8 \pm 1.8$ & $5.5 \pm 1.6$ & $5.7 \pm 1.6$ & $5.8 \pm 1.7$ & $6.2 \pm 2.0$ & $<0.001$ \\
\hline $\mathrm{TC}(\mathrm{mmol} / \mathrm{L})$ & $5.1 \pm 1.0$ & $4.8 \pm 1.0$ & $5.0 \pm 1.0$ & $5.2 \pm 0.9$ & $5.4 \pm 1.1$ & $<0.001$ \\
\hline HDL-C(mmol/L) & $1.40 \pm 0.37$ & $1.62 \pm 0.40$ & $1.45 \pm 0.34$ & $1.34 \pm 0.31$ & $1.19 \pm 0.26$ & $<0.001$ \\
\hline LDL-C(mmol/L) & $3.1 \pm 0.9$ & $2.86 \pm 0.81$ & $3.13 \pm 0.85$ & $3.26 \pm 0.85$ & $3.28 \pm 1.02$ & $<0.001$ \\
\hline $\mathrm{TG}(\mathrm{mmol} / \mathrm{L})$ & $1.6 \pm 1.0$ & $0.9 \pm 0.3$ & $1.3 \pm 0.3$ & $1.6 \pm 0.4$ & $2.7 \pm 1.5$ & $<0.001$ \\
\hline Antihypertension agents, n (\%) & $1682(50.01)$ & $324(37.07)$ & $407(49.21)$ & $452(54.33)$ & $499(60.12)$ & $<0.001$ \\
\hline LVH, n (\%) & $807(24.00)$ & $15 \mathrm{I}(17.28)$ & $177(21.40)$ & $217(26.08)$ & $262(31.57)$ & $<0.001$ \\
\hline AS, n (\%) & $1075(31.97)$ & $203(23.22)$ & $254(30.7 I)$ & $27 I(32.57)$ & $347(41.80)$ & $<0.001$ \\
\hline LEA, n (\%) & $436(12.96)$ & $99(11.33)$ & $80(9.67)$ & $110(13.22)$ & $147(|7.7|)$ & $<0.001$ \\
\hline MAU, n (\%) & $\mid 722(5 \mid .20)$ & $423(48.40)$ & $389(47.03)$ & $427(51.32)$ & $483(58.19)$ & $<0.001$ \\
\hline CKD, n (\%) & $304(9.03)$ & $7 I(8.12)$ & $56(6.77)$ & $73(8.77)$ & $104(12.53)$ & $<0.001$ \\
\hline $\mathrm{ABI}$ & $1.03 \pm 0.12$ & $1.04 \pm 0.12$ & $1.04 \pm 0.11$ & $1.02 \pm 0.13$ & $1.00 \pm 0.13$ & $<0.001$ \\
\hline $\operatorname{LVMI}\left(g / \mathrm{m}^{2}\right)$ & $87.8 \pm 28.5$ & $82.7 \pm 26.7$ & $86.7 \pm 26.7$ & $89.5 \pm 29.8$ & $92.3 \pm 29.9$ & $<0.001$ \\
\hline Cf-PWV (m/s) & $9.33 \pm 2.29$ & $8.81 \pm 2.26$ & $9.18 \pm 2.20$ & $9.42 \pm 2.17$ & $9.92 \pm 2.41$ & $<0.001$ \\
\hline eGFR $\left(\mathrm{mL} / \mathrm{min} / 1.73 / \mathrm{m}^{2}\right)$ & $85.1 \pm 20.0$ & $87.3 \pm 17.7$ & $86.5 \pm 22.7$ & $84.5 \pm 19.1$ & $82.1 \pm 19.8$ & $<0.001$ \\
\hline Log (UACR) (mg/g) & $3.46 \pm 1.13$ & $3.34 \pm 1.07$ & $3.33 \pm 1.12$ & $3.45 \pm 1.16$ & $3.70 \pm 1.12$ & $<0.001$ \\
\hline LAP & $43.5 \pm 36.0$ & $13.5 \pm 5.7$ & $28.2 \pm 4.0$ & $43.9 \pm 5.7$ & $88.1 \pm 44.6$ & $<0.001$ \\
\hline
\end{tabular}

Note: Quantitative variables are shown as mean \pm SD, and qualitative parameters are presented as numbers with the percentage in parentheses.

Abbreviations: CVD, cardiovascular disease; SBP, systolic blood pressure; DBP, diastolic blood pressure; WC, waist circumference; BMI, body mass index; FPG, fasting glucose; TC, total cholesterol; HDL-C, high-density lipoprotein cholesterol; LDL-C, low-density lipoprotein cholesterol; TG, triglycerides; LVH, left ventricular hypertrophy; AS, arterial stiffness; LEA, lower extremity atherosclerotic; MAU, micro-albuminuria; CKD, chronic kidney disease; LVMI, left ventricular mass index; cf-PWV, carotid to femoral aortic pulse wave velocity; eGFR, estimated glomerular filtration rate; UACR, urine albumin to creatinine ratio; LAP, lipid accumulation product.

$(\mathrm{OR}=1.05,95 \% \mathrm{CI}: 0.95-1.16, \mathrm{P}=0.33)$ or LEA $(\mathrm{OR}=1.04$, 95\% CI: $0.93-1.17, \mathrm{P}=0.47)$.

Table 2. Age-Sex Adjusted Partial Correlation of LAP with CVD Risk Factors

\begin{tabular}{|l|c|c|}
\hline \multirow{2}{*}{} & \multicolumn{2}{|c|}{ LAP } \\
\cline { 2 - 3 } & $\mathbf{r}$ & $\mathbf{P}$ \\
\hline BMI & 0.46 & $<0.001$ \\
SBP & 0.17 & $<0.001$ \\
DBP & 0.13 & $<0.001$ \\
FPG & 0.88 & $<0.001$ \\
TC & 0.19 & $<0.001$ \\
HDL-C & 0.03 & 0.09 \\
LDL-C & -0.44 & $<0.001$ \\
\hline
\end{tabular}

Abbreviations: LAP, lipid accumulation product; CVD, cardiovascular disease; BMI, body mass index; SBP, systolic blood pressure; DBP, diastolic blood pressure; FPG, fasting glucose; TC, total cholesterol; HDL-C, high-density lipoprotein cholesterol; LDL-C, low-density lipoprotein cholesterol.

\section{Discussion}

Based on the NSS study with 3363 Chinese communitydwelling elderly enrolled, we explored the association of LAP with TOD. LAP is associated with arterial stiffness, CKD and MAU.

Fat can be accumulated either subcutaneously or viscerally, while visceral fat accumulation may be more closely associated with metabolic disorders and predict CVD. Recent studies have shown that LAP is a simple index of visceral fat accumulation and superior to other conventional indexes. ${ }^{6,22,23}$ LAP has long been a simple and inexpensive biomarker for metabolic disorders. ${ }^{24}$ Studies have shown LAP was associated with abnormal glucose regulation ${ }^{8,14}$ and predicted type 2 diabetes mellitus to be better than $\mathrm{BMI}^{25,26}$ and $\mathrm{WC} .^{27}$ Meanwhile, LAP could more accurately diagnose metabolic syndrome in healthy men ${ }^{11,12}$ and in patients with CKD. ${ }^{28} \mathrm{We}$ 
Table 3. Univariate Logistic Regression for Risk of TOD According to LAP Quartiles

\begin{tabular}{|l|c|c|c|c|c|}
\hline & Q1 & Q2 & Q3 & Q4 & P for tread \\
\hline LVH & 1 & $1.31(1.03-1.67)$ & $1.70(1.34-2.15)$ & $2.22(1.76-2.79)$ & $<0.001$ \\
AS & 1 & $1.47(1.18-1.83)$ & $1.59(1.28-1.98)$ & $2.37(1.91-2.93)$ & $<0.001$ \\
LEA & 1 & $0.84(0.61-1.15)$ & $1.19(0.89-1.59)$ & $1.69(1.28-2.22)$ & $<0.001$ \\
MAU & 1 & $0.95(0.78-1.15)$ & $1.13(0.93-1.36)$ & $1.49(1.22-1.80)$ & $<0.001$ \\
CKD & 1 & $0.83(0.58-1.20)$ & $1.11(0.78-1.56)$ & $1.65(1.20-2.26)$ & $<0.001$ \\
\hline
\end{tabular}

Abbreviations: TOD, target organ damage; LAP, lipid accumulation product; LVH, left ventricular hypertrophy; AS, arterial stiffness; LEA, lower extremity atherosclerotic; MAU, micro-albuminuria; CKD, chronic kidney disease.

Table 4. Multivariable Logistic Regression for Risk of TOD According to LAP Quartiles

\begin{tabular}{|l|c|c|c|c|c|}
\hline & QI & Q2 & Q3 & Q4 $^{\text {P3 }}$ & $\mathbf{P}_{\text {for tread }}$ \\
\hline LVH & $\mathrm{I}$ & $1.02(0.78-1.34)$ & $1.10(0.82-1.47)$ & $1.19(0.85-1.65)$ & 0.25 \\
AS & 1 & $1.31(1.02-1.70)$ & $1.39(1.05-1.85)$ & $1.88(1.37-2.58)$ & $<0.001$ \\
LEA & 1 & $0.64(0.45-0.90)$ & $0.79(0.55-1.12)$ & $0.87(0.58-1.29)$ & 0.96 \\
MAU & 1 & $0.91(0.73-1.13)$ & $1.06(0.84-1.36)$ & $1.33(1.01-1.75)$ & 0.02 \\
CKD & 1 & $1.35(0.83-2.19)$ & $1.78(1.08-2.92)$ & $2.39(1.39-4.12)$ & $<0.001$ \\
\hline
\end{tabular}

Note: Adjusted for age, sex, hypertension, use of antihypertensive, diabetes, BMI, smoking habit, HDL-C, LDL-C, family history of premature CVD.

Abbreviations: TOD, target organ damage; LAP, lipid accumulation product; LVH, left ventricular hypertrophy; AS, arterial stiffness; LEA, lower extremity atherosclerotic; MAU, micro-albuminuria; CKD, chronic kidney disease; BMI, body mass index; HDL-C, high-density lipoprotein cholesterol; LDL-C, low-density lipoprotein cholesterol; CVD, cardiovascular disease.

found that LAP correlates with traditional biochemical risk markers. LAP may be an index closely related to metabolic disorders, but is it superior to the traditional indices is not clear.

TOD is an early warning sign for CVD. TOD mainly includes heart damage ( $\mathrm{LVH}$, Left ventricular diastolic dysfunction), vascular damage (AS, LEA), kidney

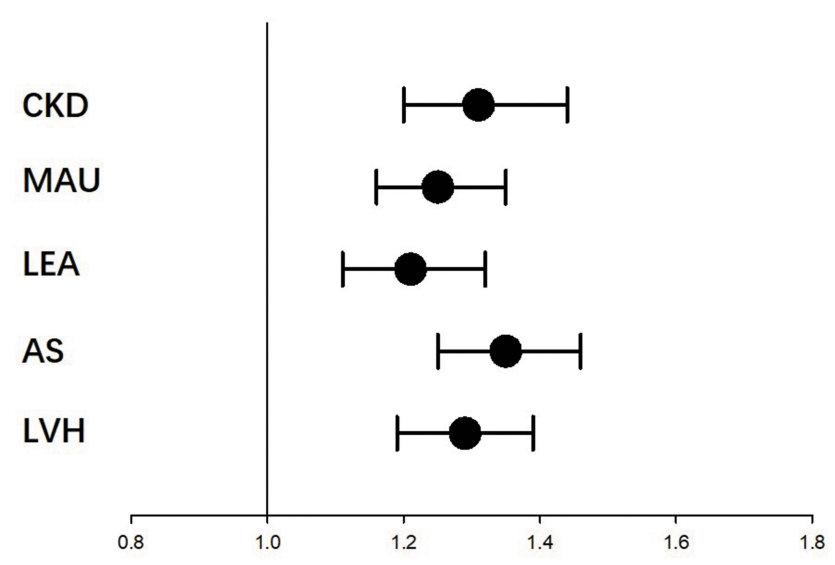

Figure I Univariate logistic regression for risk of TOD according to the I SD increasement of the LAP. For participants with I-SD increasement of LAP, they have significant added risk of LVH, AS, LEA, CKD, MAU.

Abbreviations: TOD, target organ damage; SD, standard deviation; LAP, lipid accumulation product; LVH, left ventricular hypertrophy; AS, arterial stiffness; LEA, lower extremity atherosclerotic; MAU, micro-albuminuria; CKD, chronic kidney disease. damage (MAU, CKD). It is well known that metabolic disorder is closely related to the occurrence of TOD. LVH is an important TOD that relates to lipid profiles as well. $^{29}$ Wang et al reported that non-traditional lipid profiles were associated with left ventricular geometry change in the general population of rural China. ${ }^{30}$ In our

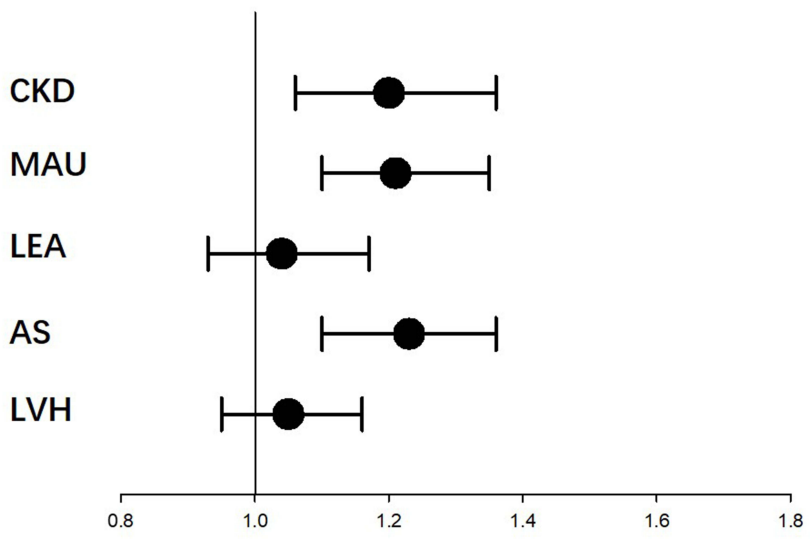

Figure 2 Multivariate logistic regression for risk of TOD according to the I SD increasement of the LAP. For participants with I-SD increasement of LAP, they have significant added risk of AS, CKD, MAU; but not LVH or LEA. Adjusted age, sex, hypertension, use of antihypertensive, diabetes, BMI, smoking habit, HDL-C, LDL$C$, family history of premature CVD.

Abbreviations: TOD, target organ damage; SD, standard deviation; LAP, lipid accumulation product; LVH, left ventricular hypertrophy; AS, arterial stiffness; LEA, lower extremity atherosclerotic; MAU, micro-albuminuria; CKD, chronic kidney disease; BMI, body mass index; HDL-C, high-density lipoprotein cholesterol; LDL-C, low-density lipoprotein cholesterol; CVD, cardiovascular disease. 
study, although the novel metabolic index, LAP was associated with LVH in univariate regression analysis, the association became non-significant after adjustment for confounding variables. This may be because metabolic disorders precede blood pressure changes and LVH is more closely associated with blood pressure.

ABI is widely used in the evaluation of peripheral artery disease. A study reported that LAP was one of the most powerful predictors of $\mathrm{ABI}<0.9$ when compared with other metabolic indices among participants with type 2 diabetes. $^{31}$ But the study did not consider other clinical parameters, such as BMI and blood pressure, which are important in peripheral artery disease. In the present study, we found that LAP was only associated with LEA in univariate regression analysis, but lost significance in multivariable logistic regression analysis.

Cf-PWV is regarded as "gold standard" of arterial stiffness. ${ }^{32}$ Metabolic components were associated with cfPWV in cross-sectional studies. ${ }^{33,34}$ A longitudinal study reported that cf-PWV progression was related to serum triglycerides, fast glucose tolerance, and BMI. ${ }^{35}$ However, many longitudinal studies showed the cf-PWV progression was determined by age and blood pressure rather than metabolic components. ${ }^{36-38}$ This may be because the sample size was relatively small and changes in PWV were not apparent at 3-4 years of follow-up. This maybe the first paper reporting the association between LAP and cf-PWV, but the relationship between LAP and cf-PWV needs more studies to prove, especially the longitudinal studies with long-term follow-up and large samples.

Besides, we also found that LAP was associated with CKD and MAU. This is consistent with the findings of previous cross-sectional studies. ${ }^{39-41}$ Kidney damage may be more sensitive to metabolic disorder. However, more cohort studies are needed to confirm this finding.

More importantly, LAP independently indicates the risks of CVD. In 2005, Kahn found that LAP better predicted CVD compared with BMI from the third National Health and Nutrition Examination Survey. ${ }^{6}$ Thereafter, more and more studies showed the predictive value of LAP for CVD. ${ }^{42,43}$ However, whether LAP performed better than other indices like BMI and WC remained contradictory. A comparative study showed that LAP was not superior over other indices. ${ }^{44}$ Meanwhile, another study presented that LAP was independently associated with mortality among CVD patients rather than BMI. $^{15}$ Thus, the relationship between LAP and CVD necessitates further illustration. In our study, an increased
LAP was associated with AS, CKD and MU, but was not superior to WC (Supplementary 1).

TOD is an early warning sign for CVD. However, to our knowledge, the relationship between LAP and TOD has not been comprehensively and systemically explored. Our study pioneeringly assessed the association between LAP and TOD, including heart, vascular and kidney, among the largely elderly Chinese population. For all this, LAP was associated with AS, CKD and MAU, but not LVH or LEA. The results are the same as previous studies. ${ }^{45}$ This may be because different organ damage has different mechanism.

There are several limitations in the present study. First, this is a cross-sectional study with relatively weak inferences about cause and effect. Second, participants are only elderly Chinese, and the conclusion should be extrapolated with caution to other populations.

\section{Conclusions}

The novel metabolic parameter LAP is significantly and independently associated with an increased risk of arterial stiffness, chronic kidney disease and micro-albuminuria in Chinese community-dwelling elderly individuals.

\section{Data Sharing Statement}

The datasets used and/or analyzed in this study are available from the corresponding authors upon reasonable request.

\section{Ethics Approval and Informed Consent}

This study was approved by the Ethics Committee of Shanghai Tenth People's Hospital and was conducted in accordance with the Declaration of Helsinki. All participants provided written informed consent.

\section{Acknowledgments}

Thanks to all the investigators and subjects who participated in the Northern Shanghai Study.

\section{Funding}

This study was financially supported by the National Nature Science Foundation of China (82170388), Clinical Research Plan of SHDC (No. SHDC2020CR1040B, SHDC2020CR5009, SHDC2020CR5015-002), Shanghai Technology Research Leader Program (21XD1434700), and the Cardiac rehabilitation fund by the International Medical Exchange Foundation (Z-2019-42-1908-3). 


\section{Disclosure}

The authors report no conflicts of interest.

\section{References}

1. Fox CS, Massaro M, Hoffmann U, et al. Abdominal visceral and subcutaneous adipose tissue compartments: association with metabolic risk factors in the Framingham Heart Study. Circulation 2007;116(1):39-48. doi:10.1161/circulationaha.106.675355

2. Preis SR, Massaro M, Robins SJ, et al. Abdominal subcutaneous and visceral adipose tissue and insulin resistance in the Framingham heart study. Obesity (Silver Spring, Md). 2010;18(11):2191-2198. doi:10.1038/oby.2010.59

3. Camhi SM, Bray GA, Bouchard C, et al. The relationship of waist circumference and BMI to visceral, subcutaneous, and total body fat: sex and race differences. Obesity (Silver Spring, Md). 2011;19 (2):402-408. doi:10.1038/oby.2010.248

4. Shah RV, Murthy VL, Abbasi A, et al. Visceral adiposity and the risk of metabolic syndrome across body mass index: the MESA Study. JACC. 2014;7(12):1221-1235. doi:10.1016/j.jcmg.2014.07.017

5. Neeland IJ, Turer T, Ayers CR, et al. Dysfunctional adiposity and the risk of prediabetes and type 2 diabetes in obese adults. JAMA. 2012;308(11):1150-1159. doi:10.1001/2012.jama.11132

6. Kahn HS. The "lipid accumulation product" performs better than the body mass index for recognizing cardiovascular risk: a populationbased comparison. BMC Cardiovasc Disord. 2005;5:26. doi:10.1186/ 1471-2261-5-26

7. Wiltgen D, Benedetto IG, Mastella LS, Spritzer PM. Lipid accumulation product index: a reliable marker of cardiovascular risk in polycystic ovary syndrome. Hum Reprod. 2009;24(7):1726-1731. doi:10.1093/humrep/dep072

8. Oh J-Y, Sung Y-A, Lee HJ. The lipid accumulation product as a useful index for identifying abnormal glucose regulation in young Korean women. Diabetic Med. 2013;30(4):436-442. doi:10.1111/ dme. 12052

9. Kahn HS. The lipid accumulation product is better than BMI for identifying diabetes: a population-based comparison. Diabetes Care. 2006;29(1):151-153. doi:10.2337/diacare.29.1.151

10. Tellechea ML, Aranguren FA, Martinez-Larrad M, Serrano-Rios M, Taverna MJ, Frechtel GD. Ability of lipid accumulation product to identify metabolic syndrome in healthy men from Buenos Aires. Diabetes Care. 2009;32(7):e85. doi:10.2337/dc08-2284

11. Taverna MJT, Martínez-Larrad MT, Frechtel GD, Serrano-Ríos M. Lipid accumulation product: a powerful marker of metabolic syndrome in healthy population. Eur $J$ Endocrinol. 2011;164 (4):559-567. doi:10.1530/eje-10-1039

12. Ray L, Ravichandran K, Nanda SK. Comparison of lipid accumulation product index with body mass index and waist circumference as a predictor of metabolic syndrome in Indian Population. Metab Syndr Relat Disord. 2018;16(5):240-245. doi:10.1089/met.2017.0119

13. Brahimaj A, Rivadeneira F, Muka T, et al. Novel metabolic indices and incident type 2 diabetes among women and men: the Rotterdam Study. Diabetologia. 2019;62(9):1581-1590. doi:10.1007/s00125019-4921-2.

14. Wehr E, Gruber H-J, Giuliani A, Möller R, Pieber TR, ObermayerPietsch B. The lipid accumulation product is associated with impaired glucose tolerance in PCOS women. $J$ Clin Endocrinol Metab. 2011;96(6):E986-90. doi:10.1210/jc.2011-0031

15. Ioachimescu AG, Brennan DM, Hoar BM, Hoogwerf BJ. The lipid accumulation product and all-cause mortality in patients at high cardiovascular risk: a PreCIS database study. Obesity (Silver Spring, Md). 2010;18(9):1836-1844. doi:10.1038/oby.2009.453

16. Schunkert H. Obesity and target organ damage: the heart. Int J Obes Rel Metab Disord. 2002;S15-20. doi:10.1038/sj.ijo.0802214
17. De Jong PE, Verhave JC, Pinto-Sietsma SJ, Hillege HL. Obesity and target organ damage: the kidney. Int J Obes Rel Metab Disord. 2002; S21-4. doi:10.1038/sj.ijo.0802213

18. Palatini P, Saladini F, Mos L, et al. Obesity is a strong determinant of hypertensive target organ damage in young-to-middle-age patients. Int J Obes. 2013;37(2):224-229. doi:10.1038/ijo.2012.32.

19. Fassini G, Conti S, Moltrasio M, et al. Concomitant cryoballoon ablation and percutaneous closure of left atrial appendage in patients with atrial fibrillation. Europace. 2016;18(11):1705-1710. doi:10.1093/europace/euw007

20. Ji H, Xiong J, Yu S, et al. Northern Shanghai Study: cardiovascular risk and its associated factors in the Chinese elderly-a study protocol of a prospective study design. BMJ Open. 2017;7(3):e013880. doi:10.1136/bmjopen-2016-013880.

21. Beck GJ, Berg RL, Coggins C, et al. Design and statistical issues of the modification of diet in renal disease trial. The Modification of Diet in Renal Disease Study Group. Control Clin Trials. 1991;12 (5):566-586. doi:10.1016/0197-2456(91)90069-x

22. Roriz AKC, Passos LCS, de Oliveira CC, et al. Evaluation of the accuracy of anthropometric clinical indicators of visceral fat in adults and elderly. PLoS One. 2014;9(7):e103499. doi:10.1371/journal. pone. 0103499

23. Ganguly S, Ray L, Kuruvila S, Nanda SK, Ravichandran K. Lipid accumulation product index as visceral obesity indicator in psoriasis: a case-control study. Indian J Dermatol. 2018;63(2):136-140. doi:10.4103/ijd.IJD_315_16.

24. Rotter I, Rył A, Szylińska A, Pawlukowska W, Lubkowska A, Laszczyńska M. Lipid Accumulation Product (LAP) as an index of metabolic and hormonal disorders in aging men. Exp Clin Endocrinol Diabetes. 2017;125(3):176-182. doi:10.1055/s-0042-116071.

25. Bozorgmanesh M, Hadaegh F, Azizi F. Diabetes prediction, lipid accumulation product, and adiposity measures; 6-year follow-up: Tehran lipid and glucose study. Lipids Health Dis. 2010;9(1):45. doi:10.1186/1476-511x-9-45

26. Tian T, Pei H, Chen Z, et al. Comparison of lipid accumulation product and body mass index as indicators of diabetes diagnosis among 215,651 Chinese adults. Peer J. 2020;8:e8483. doi:10.7717/ peerj. 8483

27. Wang Z, He S, Chen X. Capacity of different anthropometric measures to predict diabetes in a Chinese population in southwest China: a 15-year prospective study. Diabetic Med. 2019;36(10):1261-1267. doi:10.1111/dme.14055

28. Biyik Z, Guney IJ. Lipid accumulation product and visceral adiposity index: two new indices to predict metabolic syndrome in chronic kidney disease. Eur Rev Med Pharmacol Sci. 2019;23(5):2167-2173. doi:10.26355/eurrev_201903_17262.

29. Wang H, Li Z, Guo X, et al. The impact of nontraditional lipid profiles on left ventricular geometric abnormalities in general Chinese population. BMC Cardiovasc Disord. 2018;18(1):88. doi:10.1186/s12872-018-0829-x

30. Wang H, Sun Y, Li Z, et al. Gender-specific contribution of cardiometabolic index and lipid accumulation product to left ventricular geometry change in general population of rural China. $B M C$ Cardiovasc Disord. 2018;18(1):62. doi:10.1186/s12872-018-0798-0.

31. Wung C, Lee M, Wu P, Huang J, Chen S. Obesity-related indices are associated with peripheral artery occlusive disease in patients with Type 2 diabetes mellitus. J Pers Med. 2021;11(6):533. doi:10.3390/ jpm11060533

32. Laurent S, Cockcroft J, Van Bortel L, et al. Expert consensus document on arterial stiffness: methodological issues and clinical applications. Eur Heart J. 2006;27(21):2588-2605. doi:10.1093/eurheartj/eh1254

33. Lopes-Vicente WR, Rodrigues S, Cepeda FX, et al. Arterial stiffness and its association with clustering of metabolic syndrome risk factors. Diabetol Metab Syndr. 2017;9:87. doi:10.1186/s13098-017-0286-1 
34. Topouchian J, Labat C, Gautier S, et al. Effects of metabolic syndrome on arterial function in different age groups: the Advanced Approach to Arterial Stiffness study. J Hypertens. 2018;36 (4):824-833. doi:10.1097/hjh.0000000000001631

35. Zachariah J, Rong J, Larson M, et al. Metabolic predictors of change in vascular function: prospective associations from a community-based cohort. Hypertension (Dallas, Tex:1979). 2018;71 (2):237-242. doi:10.1161/hypertensionaha.117.10054

36. Meani P, Maloberti A, Sormani P, et al. Determinants of carotid-femoral pulse wave velocity progression in hypertensive patients over a 3.7 years follow-up. Blood Press. 2018;27(1):32-40. doi:10.1080/08037051.2017.1378069

37. Maloberti A, Rebora P, Andreano A, et al. Pulse wave velocity progression over a medium-term follow-up in hypertensives: focus on uric acid. J Clin Hypertens. 2019;21(7):975-983. doi:10.1111/ jch. 13603

38. Maloberti A, Bruno R, Facchetti R, et al. THE role of metabolic syndrome in blood pressure control and pulse wave velocity progression over a 3.5 years in treated hypertensive PATIENTS. Eur J Intern Med. 2020;76:107-109. doi:10.1016/j.ejim.2020.02.005

39. Dai D, Chang Y, Chen Y, et al. Visceral adiposity index and lipid accumulation product index: two alternate body indices to identify chronic kidney disease among the Rural Population in Northeast China. Int J Environ Res Public Health. 2016;13:12. doi:10.3390/ ijerph13121231
40. Zhang K, Li Q, Chen Y, Wang N, Lu Y. Visceral adiposity and renal function: an observational study from SPECT-China. Lipids Health Dis. 2017;16(1):205. doi:10.1186/s12944-017-0597-0

41. Liu Y, Wang Y, Wang J, et al. Lipid accumulation product is associated with urinary albumin-creatinine ratio in Chinese prediabetic population: a report from the REACTION Study. Diabetes Metab Syndr Obes. 2021;14:2415-2425. doi:10.2147/dmso.S310751

42. Jx N, Mb C, de Sousa RML, et al. Importance of lipid accumulation product index as a marker of CVD risk in PCOS women. Lipids Health Dis. 2015;14:62. doi:10.1186/s12944-015-0061-y

43. Wehr E, Pilz S, Boehm BO, März W, Obermayer-Pietsch B. The lipid accumulation product is associated with increased mortality in normal weight postmenopausal women. Obesity (Silver Spring, Md). 2011;19(9):1873-1880. doi:10.1038/oby.2011.42

44. Hosseinpanah F, Barzin M, Mirbolouk M, et al. Lipid accumulation product and incident cardiovascular events in a normal weight population: Tehran Lipid and Glucose Study. Eur J Prev Cardiol. 2016;23 (2):187-193. doi:10.1177/2047487314558771.

45. Maloberti A, Bombelli M, Vallerio P, et al. Metabolic syndrome is related to vascular structural alterations but not to functional ones both in hypertensives and healthy subjects. Nutri Metabol Cardiovasc Dis. 2021;31(4):1044-1052. doi:10.1016/j.numecd.2020.11.011
Clinical Interventions in Aging

\section{Publish your work in this journal}

Clinical Interventions in Aging is an international, peer-reviewed journal focusing on evidence-based reports on the value or lack thereof of treatments intended to prevent or delay the onset of maladaptive correlates of aging in human beings. This journal is indexed on PubMed Central, MedLine, CAS, Scopus and the Elsevier

\section{Dovepress}

Bibliographic databases. The manuscript management system is completely online and includes a very quick and fair peer-review system, which is all easy to use. Visit http://www.dovepress.com/ testimonials.php to read real quotes from published authors. 